\title{
A Novel Framework for Supporting Mobile Object Self-Localization via Emerging Artificial Intelligence Tools
}

\author{
Alfredo Cuzzocrea* \\ iDEA Lab, University of Calabria, Rende, Italy \& LORIA, Nancy, France \\ alfredo.cuzzocrea@unical. it \\ Luca Camilotti \\ University of Trieste, Trieste, Italy \\ camilottidunits.it \\ Enzo Mumolo \\ University of Trieste, Trieste, Italy \\ mumolo@units.it
}

\begin{abstract}
This paper focuses the attention on the problem of supporting mobile object self-localization. The proposed algorithm estimates the distance from the camera lens to the center of the landmarks using only ceiling vision. Localization can be easily obtain from such distance estimations. Projections are geometrically described and the distance estimation is based on the pixels mapping information obtained by a two-dimensional dynamic programming algorithm (2D-DPA).
\end{abstract}

\section{Introduction}

Self-localization of mobile objects is a fundamental requirement for autonomy (e.g., [25]). Mobile objects can be for example a mobile service robot, a motorized wheelchair, a mobile cart for transporting tasks or similar. Selflocalization represents as well a necessary feature to develop systems able to perform autonomous movements such as navigation tasks. Self-localization is based upon reliable information coming from sensor devices situated on the mobile objects. There are many sensors available for that purpose. The early devices for positioning are rotary encoders. If the encoders are connected to wheels or legs movement actuators, relative movements of the mobile object during its path [1] can be measured. Then, mobile object positioning can be obtained with dead-reckoning approaches. Dead reckoning [1] is still widely used for mobile robot positioning estimation. It is also true that dead-reckoning is quite unreliable for long navigation tasks, because of accumu-

${ }^{*}$ This research has been made in the context of the Excellence Chair in Computer Engineering - Big Data Management and Analytics at LORIA, Nancy, France lated error problems. Other popular sensor devices for selflocalization are laser or sonar based range finder devices and inertial measurement devices. In outside scenarios the most popular approaches are based on Global Positioning System (GPS). Due to the importance of self-localization, many other solutions for indoor environment have been proposed so far with different cost and accuracy characteristics. For example the Ultra Wide Band radio signal indoor localization systems [2], or the Bluetooth-based angle of arrival radio devices [3], or a combination of them. However these systems have serious limitations in cost and reliability, respectively. Another important type of sensors which may be used for cost effective self-localization are the CCD cameras, which require computer vision algorithms for localization such as for example visual odometry, [14]. Mobile objects vision self-localization is currently an open research field [5] and an increasing number of new methods are continuously proposed. As a matter of fact we have to consider that self-localization of mobile objects requires centimeter-level accuracy and Computer Vision is one of the most cost-effective techniques able to reach that accuracy. Consequently, some surveys of Computer Vision based selflocalization techniques appeared recently in the literature, [6].

In this paper we describes a novel Computer Vision algorithm for estimating the distance from the camera lens to the center of ceiling landmarks with circular shape using a monocular low cost webcam. From the distance, mobile object localization approaches can be easily developed and a simple example is provided in this paper. The images of the ceiling landmarks are projected on the image plane of the camera. The projection is analytical described, but the projections distortions, which may arise especially when low cost devices are used, may affect the results. To take into account the projection distortions in order to obtain a better precision of the results, we use an approximation of

DOI reference number: 10.18293/DMSVIVA2021-019 
the two-dimensional dynamic programming (2D-DPA) algorithm [4] which finds a sub-optimal mapping between the image pixels of the ceiling landmarks and the image plane pixels of the projected landmarks. Since optimum 2D-DPA is NP-complete, in fact, many approximations have been developed. For example, the 2D-DPA technique described by Levin and Pieraccini in [16] has an exponential complexity in the image size, while Uchida and Sakoe describe in [15] a Dynamic Planar Warping technique with a complexity equal to $O\left(N^{3} 9^{N}\right)$. Lei and Govindaraju propose in [17] a Dynamic Planar Warping approximation with a complexity of $O\left(N^{6}\right)$. However each approximation has some limitation in terms of continuity of the mapping. In this paper we use a approximation of the optimum 2D-DPA with a complexity of $O\left(N^{4}\right)$ [13] which is implemented on a GPU to obtain real-time performance. When the landmark is far from the camera or if the environments has low lighting conditions, an high quantization noise may arise in acquired images. However the algorithm we describe in this paper is particularly robust against noise due especially to the use of two-dimension DPA.

Another interesting aspect of our research consists in considering the emerging integration of these topics with the innovative big data trend (e.g., [7, 8, 9]). Here, the main research perspective is that to take into account the well-known $3 \mathrm{~V}$ model of big data, including volume, velocity and variety.

The extended version of this paper appears in [22].

\section{Problem Formulation}

We show in Figure 1 a mobile object in an indoor environment. The movable object is equipped with a camera set tilted towards the ceiling at an angle $\varphi$. We call $h$ the distance between the camera and the ceiling. Moreover in Figure 2 the horizontal and vertical angles of view of the camera, called $\theta_{x}$, and $\theta_{y}$ respectively, are highlighted.

The direction towards which the camera is oriented is shown with the 'Camera Direction' arrow. The ceiling landmark is shown in Figure 1 with a segment with a greater thickness and the image plane of the camera is shown with a segment orthogonal to the camera direction. The ceiling landmark is projected to the landmark on the image plane. The visual landmarks positioned on the ceiling used in this approach are the lighting holders shown as that shown in Figure 3. We choose landmarks with isotropic shapes on the plane because in this way the distortion components due to image rotation can be eliminated. The simpler isotropic shape is the circle. As shown in Figure 3, the lines of pixels on the image plane are all parallel to the reference abscissa on the ceiling plane regardless of the angle of the camera with respect to the landmark. It is important to remark that each landmark must be distinguishable from the others and its co-

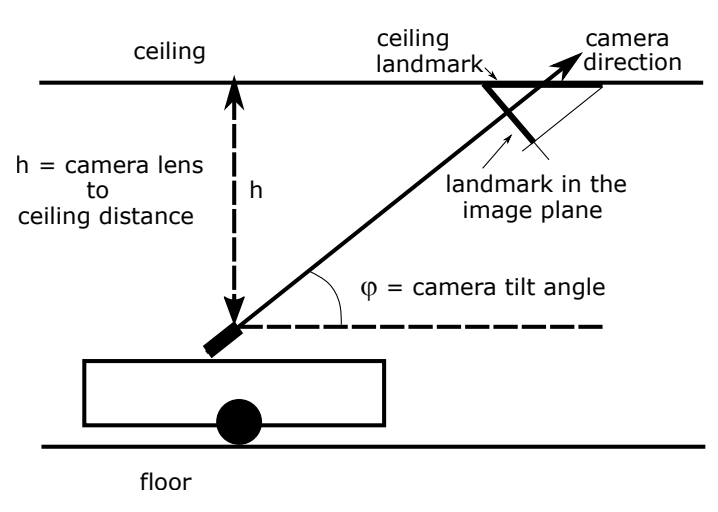

Figure 1. A mobile object with a camera on it, tilted toward the ceiling.

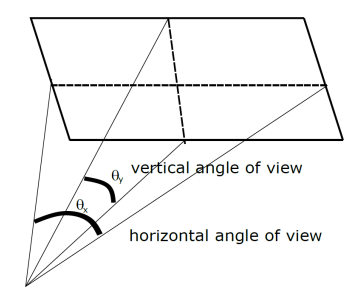

Figure 2. The horizontal and vertical angles of view of the camera.

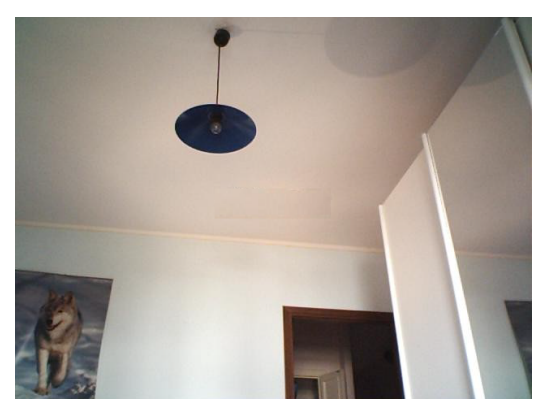

Figure 3. An example of the circular lamp holder used in this paper

ordinate in the global reference system must be known. A schematic representation of a mobile object and some landmarks with the orthonormal reference system centered on the camera lens is shown in Figure 4. The reference abscissa changes dynamically in relation to the direction of the focal axis. The reference abscissa, in fact, is always normal to the focal axis and at the same time it is parallel to the horizon.

The landmarks must be distinguishable from each other. There are many possible solutions for making the landmarks distinct. A simple possibility is to paint each holder with a different color. More recently, the characteristic fre- 


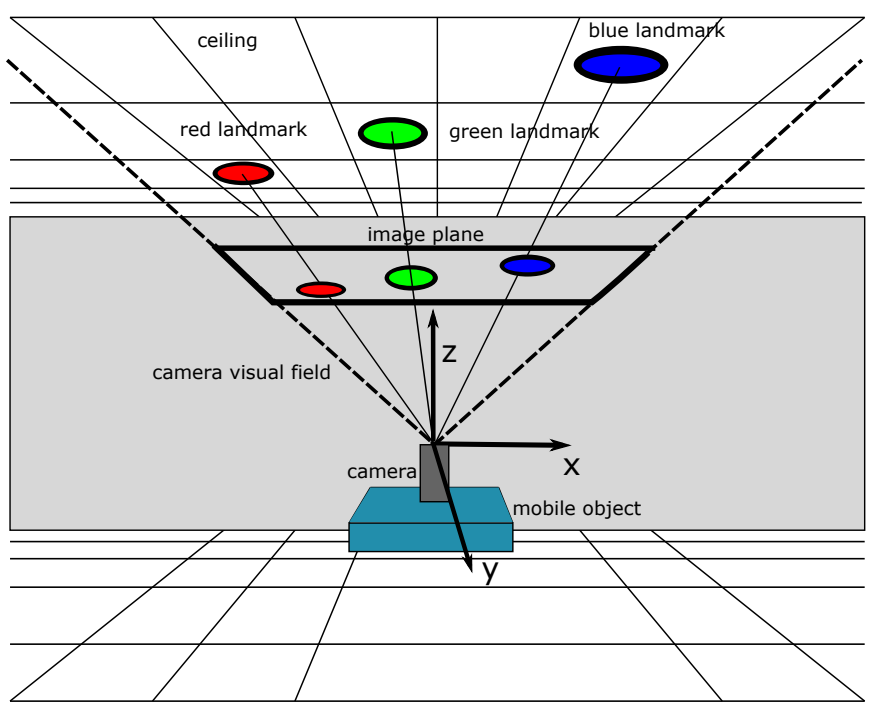

Figure 4. Schematic representation of orthonormal reference system, landmarks and image plane.

quency of fluorescent lights has been used, for instance in [11]. In this paper we used the simplest solution, namely we painted adjacent lamp holders with different colors. For this reason the landmarks in Figure 4 are represented with different colors, where for simplicity the three circular landmarks positioned on the ceiling are colored in red, blue and green. Figure 4 shows that the landmarks which fall within the visual field of the camera are projected onto the image plane of the camera. Of course we know in advance the physical position of each landmark in the global reference system. On the other hand the landmark colours can be detected using well known computer vision techniques.

\section{Intelligent Transformations}

The projective transformation is the linear transformation of coordinates reported in (1).

$$
p^{\prime}=T p
$$

where $p$ represents a generic point in space expressed in homogeneous coordinates, relative to the orthonormal reference system $S$ described by the quadruple $(O, \hat{i}, \hat{j}, \hat{k})$. The projected point $p^{\prime}$ is expressed in coordinates relative to the reference system $S^{\prime}$ described by the quadruple $\left(O^{\prime}, \hat{i}^{\prime}, \hat{j}^{\prime}, \hat{k}^{\prime}\right)$, where $\hat{i}^{\prime}=\hat{i}, \hat{j}^{\prime}$ has the direction of the segment $\overline{M Q}$ and $\hat{k}^{\prime}$ has the direction of the normal to the segment $\overline{M Q}$.

Since $p$ is expressed with the three components $\left(x_{p}, y_{p}, z_{p}\right)$ and $p^{\prime}$ has the three components $\left(x_{p^{\prime}}, y_{p^{\prime}}, z_{p^{\prime}}\right)$, eq. (1) can be also written as follows

$$
\left(\begin{array}{l}
x_{p^{\prime}} \\
y_{p^{\prime}} \\
z_{p^{\prime}}
\end{array}\right)=T\left(\begin{array}{l}
x_{p} \\
y_{p} \\
z_{p}
\end{array}\right)
$$

Such a transformation maintains the properties of collinearity, that is, the points which in $S$ belong to a line, are aligned in a line also in S'. However, projective transformation may not be defined for every point of $S$, in the sense that some points could be mapped in S' at infinity.

Let us view Figure 4 from the left side, that is the $y-z$ plane of the orthonormal reference system which has its origin coinciding with the center of the camera lens. This plane is highlighted in Figure 5, where the ceiling is at $z=h$, and the field of view of the camera is shown with points $M$ and $E$. Let us assume that a landmark falls within the vertical angle of view. Then, the center of the landmark is the point $C$. On the other hand, if we view Figure 4 from the front side, that is the $x-z$ plane, we obtain the system shown in Figure 6. Of course the camera image plane, which is the plane normal to the focal axis in Figure 4, is shown with the segment $M-Q$ in Figure 5 and segment $G-I$ in Figure 6.

Suppose we fix a point $P$ on the ceiling. If the point falls within the field of view of the camera it is shown as $P$ in Figure 5. Let $\left(p_{x}, p_{y}, p_{z}\right)$, with $p_{z}=h$, be the coordinates of $P$. The point $P$ is projected to the image plane of the camera to the point $P^{\prime}$, which has coordinates $\left(x_{p^{\prime}}, y_{p^{\prime}}, z_{p^{\prime}}\right)$. Also the center of the landmark in Figure 5 is projected to the point $C^{\prime}$ and the segment $M-E$ is projected to the segment $M-Q$ in the image plane. In

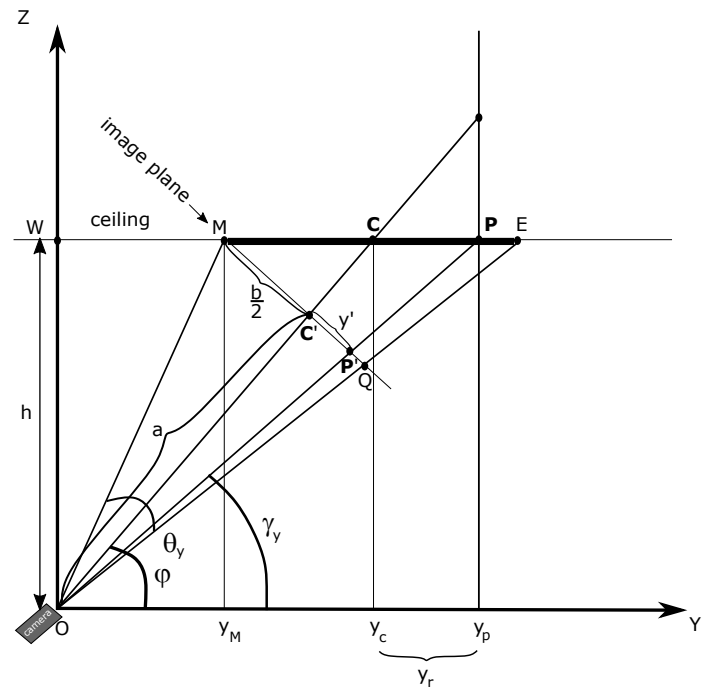

Figure 5. Plane $y-z$ in orthonormal reference system 


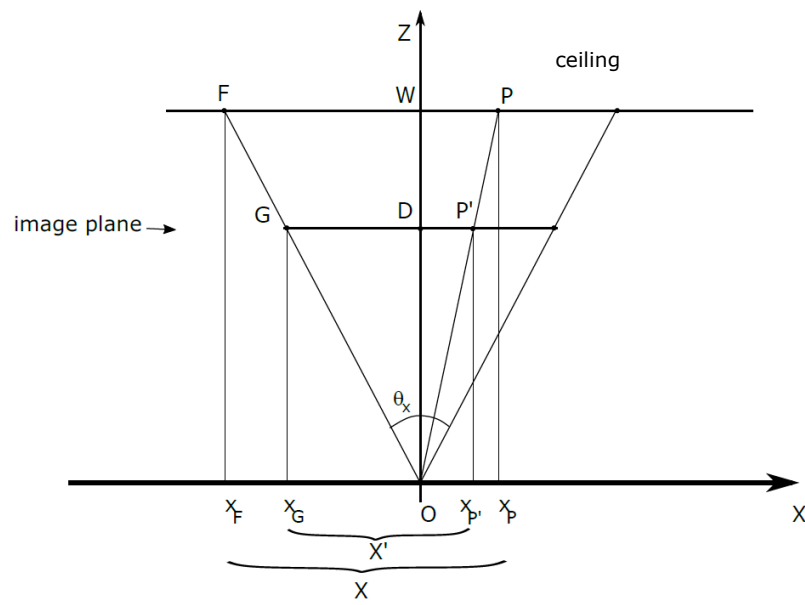

Figure 6. Plane $x-z$ in orthonormal reference system

this model, the focal distance of the device or other characteristic parameters are not taken into account. It is in fact a purely ideal model, which has the only purpose of deriving the relations that define the projective transformation from the orthonormal system whose origin coincides with the center of the camera lens to the image plane system. The latter is chosen independently of the characteristics of the camera. With reference to the Figures 5 and 6, we introduce the following geometric variables characteristic of the problem.

$$
\Phi=\varphi+\frac{\theta_{y}}{2}-\frac{\pi}{2}
$$

- The distance $a$ from the origin to the barycenter of the landmark projected on the image plane:

$$
a=\overline{O C^{\prime}}=\frac{h}{\sin (\varphi)}-h\left(\tan (\varphi)+\frac{1}{\tan (\varphi)}\right) \cos (\varphi)
$$

- The abscissa of the point $\mathrm{P}^{\prime}$ on the image plane:

$$
\frac{b}{2}=\overline{M C^{\prime}}=\overline{C^{\prime} Q}=h\left(\tan (\varphi)+\frac{1}{\tan (\varphi)} \sin (\varphi)\right)
$$

Moreover, we define the following two variables:

$$
G=-h(\tan \Phi \tan \varphi+1)
$$

and

$$
F=h(\tan \varphi-h \tan \Phi)
$$

We remark that the following considerations are based on three coordinate systems, namely an orthonormal reference system centered on the camera lens, shown in Figure 4, an orthonormal reference system on the image plane and a system on the ceiling plane which is simply translated by $h$ with respect to that centered on the camera lens. In general, points on the systems centered on the camera lens and on the ceiling are denoted with a capital letter, such as $\mathbf{P}$, while that on the image plane of the camera are denoted with a capital letter plus an apex such as $\mathbf{P}^{\prime}$. In this case, $\mathbf{P}$ ' is the $\mathbf{P}$ point projected on the image plane. If we look at the landmark seen from the orthonormal reference system centered on the camera lens, its barycenter is located at $\left(x_{c}, y_{c}\right)$. A generic point on the ceiling has coordinate $(x, y)$ and the same point projected on the image plane is $\left(x^{\prime}, y^{\prime}\right)$. The coordinates of a generic point on the landmark is given relative to its barycenter: $\left(x=x_{c}+x_{r}\right)$ and $\left(y=y_{c}+y_{r}\right)$. According to Figures 6 and 5 the offsets $x_{r}, y_{r}$ are projected to the image plane in $x^{\prime}, y^{\prime}$.

Assume now we have an optimum mapping between images. In other words, assume that, having two images $A$ and $B, A=\{a(i, j) \mid i, j=1, \ldots, N\}$ and $B=\{b(u, v) \mid u, v=$ $1, \ldots, M\}$, we can estimate the mapping function

$$
F(i, j)=\left[\begin{array}{l}
u \\
v
\end{array}\right]=\left[\begin{array}{l}
x(i, j) \\
y(i, j)
\end{array}\right]
$$

which maps each pixel $(i, j)$ of one image to the pixel $(u, v)$ of the other image such that the difference between the two images is minimized, as shown in (9).

$$
\min \sum \sum\|a(i, i)-b(u, v)\|
$$

where $u=x(i, j)$ and $v=y(i, j)$. Such mapping is performed through a two dimensional Dynamic Programming operation [15]. 2D-DPA is the base of image matching algorithms called Elastic Image Matching. Unfortunately, the Elastic Image Matching operation is NP-complete [19]. For this reason we devise an approximation which reduces the 2D-DPA operation complexity to $O\left(N^{4}\right)$, as described below.

The barycenter of the landmarks, $\left(x_{c}, y_{c}\right)$, are estimated using the following Proposition. By measuring the abscissa and ordinate $\left(x^{\prime}, y^{\prime}\right)$ of a generic point on the landmark projected on the image plane we can estimate the coordinate $\left(x_{c}, y_{c}\right)$ of the ceiling landmark using the following equations:

$$
\begin{array}{r}
x_{c}=\frac{h \cos \left(\varphi-\gamma_{y}\right)\left(x^{\prime}-g\right)}{a \sin \left(\gamma_{y}\right)}+g-x_{r} \\
y_{c}=\frac{a G+a y_{r} \tan (\varphi)-\left(y^{\prime}-\frac{b}{2}\right)\left(y_{r}+F\right)}{y^{\prime}-\frac{b}{2}-a \tan (\varphi)}
\end{array}
$$

A different estimation of the coordinates of the landmark barycenter is obtained for all the points $\boldsymbol{P}$ inside the landmarks. A sequence of barycenter coordinates $\boldsymbol{x}_{\boldsymbol{c}}, \boldsymbol{y}_{\boldsymbol{c}}$ are 
thus obtained, of which we compute the expected value. The algorithm is thus sequentially divided into two parts: estimation of $\mathbf{E}\left(\boldsymbol{x}_{\boldsymbol{c}}\right)$ and $\mathbf{E}\left(\boldsymbol{y}_{\boldsymbol{c}}\right)$ by measuring the dimension $\boldsymbol{y}^{\prime}$ and $\boldsymbol{x}^{\prime}$ of the distorted image on the image plane.

The distance from the camera lens and the landmark in the ceiling reference system is thus the following:

$$
d=\sqrt{\left.\mathrm{E}\left(x_{c}\right)^{2}+\mathrm{E}\left(y_{c}\right)^{2}\right)}
$$

with reference to Figures 6 and 5 , where $C=\left(x_{c}, y_{c}, z_{c}\right)$ is the barycenter of the landmark in the reference system $(\boldsymbol{O}, \boldsymbol{i}, \boldsymbol{j}, \boldsymbol{k})$. We obtain he sub-optimal correspondence, pixel by pixel, between a reference image and a distorted image by means of approximated two dimensional dynamic programming, . Our algorithm therefore uses the deformation of the image to derive the distance of the landmark, i.e. it is intended to determine how the perspective has distorted the image.

The coordinates of the barycenter of the ceiling landmarks are obtained using the coordinate $\boldsymbol{x}^{\prime}$ measured on the image plane and $\boldsymbol{x}_{\boldsymbol{r}}$ using the mapping function, and in terms of $\boldsymbol{y}^{\prime}$ and $\boldsymbol{y}_{\boldsymbol{r}}$. Clearly $\left(\boldsymbol{x}_{\boldsymbol{r}}, \boldsymbol{y}_{\boldsymbol{r}}\right)$ and $\left(\boldsymbol{x}^{\prime}, \boldsymbol{y}^{\prime}\right)$ are both known because they are derived from the coordinates of the pixels in the pattern and in the test images respectively. What associates the two pixels is the mapping relationship described in (8) obtained by 2D-DPA.

The characteristic that differentiates the algorithms present in the literature from the one developed in this paper is the statistical character of the obtained estimate. The algorithm based on dynamic programming is able to calculate a position estimate for each single pair of associated pixels from the mapping. The advantage is that a large number of points are used, which contribute to the calculation of the average distance value. This makes the estimate more truthful, especially when the landmark is very distant, which results in a smaller image and a greater quantization error.

\section{2D-DPA-Based Image Mapping Technique}

For the sake of coherence with what we write below, we repeat now the mapping considerations summarized above about images $\boldsymbol{A}$ and $\boldsymbol{B}$ using instead images $\boldsymbol{X}$ and $\boldsymbol{Y}$. Given the two images, $\boldsymbol{X}=\{\boldsymbol{x}(\boldsymbol{i}, \boldsymbol{j})\}$ and $\boldsymbol{Y}=\{\boldsymbol{y}(\boldsymbol{u}, \boldsymbol{v})\}$, the mapping of one image to the other is represented by the operation

$$
D(X, Y)=\min \sum_{i=1}^{N} \sum_{j=1}^{N}\|x(i, j)-y(u, v)\|
$$

where $\boldsymbol{u}=\boldsymbol{x}(\boldsymbol{i}, \boldsymbol{j}), \boldsymbol{v}=\boldsymbol{y}(\boldsymbol{i}, \boldsymbol{j})$ is the mapping function between the pixels of $\boldsymbol{X}$ and $\boldsymbol{Y}$. The quantity $\boldsymbol{D}(\boldsymbol{X}, \boldsymbol{Y})$ gives a distance between the image $\boldsymbol{X}$ and the optimally deformed $\boldsymbol{Y}$, the optimal warping function $\boldsymbol{x}(\boldsymbol{i}, \boldsymbol{j}), \boldsymbol{y}(\boldsymbol{i}, \boldsymbol{j})$ gives an interpretation of the image $\mathrm{X}$ according to the generation model $\mathrm{Y}$.

Given the $i$-th row of the $X$ image and the $j$-th row of the $\boldsymbol{Y}$ images, namely $\boldsymbol{Y}_{\boldsymbol{j}}=\left(\boldsymbol{y}_{\boldsymbol{j}, \mathbf{1}}, \boldsymbol{y}_{\boldsymbol{j}, \mathbf{2}}, \ldots, \boldsymbol{y}_{\boldsymbol{j}, \boldsymbol{N}}\right)$, $X_{i}=\left(x_{i, 1}, x_{i, 2}, \ldots, x_{i, N}\right)$ respectively, the distance between the two rows is obtained by applying a 1D-DPA [18] for finding a warping among the two rows as described in (13). Here the map $\boldsymbol{M}^{\prime}$ is, say, over $(\boldsymbol{n}, \boldsymbol{m})$ coordinates, so that $M_{l}^{\prime}=\left(\left(i_{l}, n_{l}\right),\left(j_{l}, m_{l}\right)\right)$.

$d\left(X_{i}, Y_{j}\right)=\frac{\min _{M^{\prime}} \sum_{l=1}^{M^{\prime}} d\left(M_{l}^{\prime}\right)}{M^{\prime}}=\frac{\min _{M^{\prime}} \sum_{l=1}^{M^{\prime}}\left\|x_{i_{l}, n_{l}}-y_{j_{l}, m_{l}}\right\|}{\underset{(13)}{2 N}}$

Finally, the distance between the two images is obtained by (14). In this case the map $\overline{M^{\prime}}$ is between all the rows of $\mathrm{X}$ and Y. As before, $\left|\overline{M^{\prime}}\right|$ is the length of the path.

$$
\begin{gathered}
D(X, Y)=\frac{\frac{\min }{M^{\prime}} \sum_{k} d\left(\overline{M^{\prime}}{ }_{k}\right)}{\left|\overline{M^{\prime}}\right|}= \\
=\frac{\min _{M^{\prime}} \sum_{k} d\left(X_{i_{k}}, Y j_{k}\right)}{\left|\overline{M^{\prime}}\right|}=\frac{\min _{M^{\prime}} \sum_{k} \frac{\min _{M^{\prime}} \sum_{l=1}^{\left|M^{\prime}\right|} d\left(M_{l}^{\prime}\right)}{2 N}}{2 N}= \\
=\frac{\min _{M^{\prime}}\left\{\sum_{k} \min _{M^{\prime}} \sum_{l=1}^{\left|M^{\prime}\right|}\left\|x_{i, n_{l}}-y_{j, m_{l}}\right\|\right\}}{4 N^{2}}
\end{gathered}
$$

Let us assume that the images are of equal size, that is $N \times N$ pixels. Then the length of the optimum path between the two images is equal to $2 N$. The local distances in each point of this path is obtained with other 1DDPA with paths of length $2 N$. The total length is the sum of $2 N$ along the $2 N$ long path, giving $4 N^{2}$ at the denominator. The complexity of the described operation is $O\left(N^{2} N^{2}\right)=O\left(N^{4}\right.$ where $N$ is the image dimension.

The algorithm described in this paper is summarized in the following Algorithm. The inputs of the algorithm are the two gray-scale images $\boldsymbol{i} \boldsymbol{m} \boldsymbol{g}_{\boldsymbol{A}}$ and $\boldsymbol{i} \boldsymbol{m} \boldsymbol{g}_{\boldsymbol{B}}$ which are the landmark on the image plane and on the ceiling respectively. We perform the 2D-DPA algorithm on these two images to obtain the mapping function as result. the mapping function is represented with a linked list where each node is the map related to the two pixels. The function $\operatorname{get}()$ give as result the value of the pixel on the image indicated as input and is used to get the values of the two pixels linked by the map on the two landmark images. To decide if the pixel is a landmark pixel or not, we consider their gray levels. The landmarks have a lower values with respect to the environment and thus if the pixel values is less then a thereshold, the pixel is a landmark pixel. 


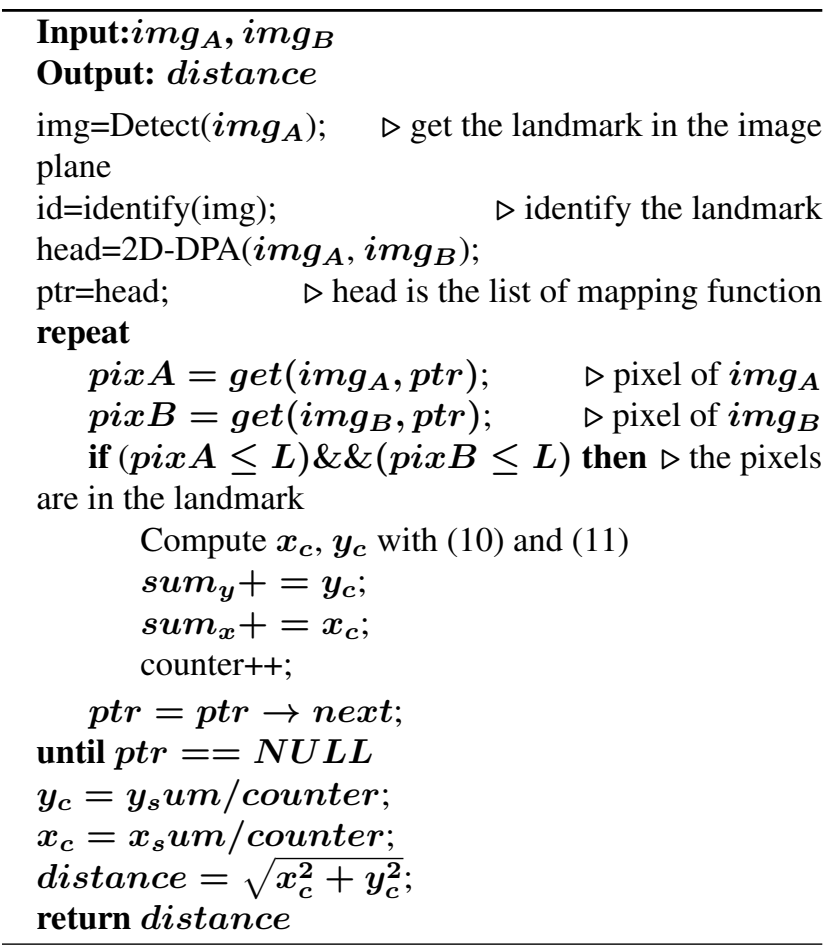

\section{Conclusions and Future Work}

In this paper we present an algorithm to measure the distance of a mobile object to the lightning lamps used as ceiling landmarks in indoor environment. The algorithm has many attractive features, mainly the accuracy, which is better than many other visual-based algorithms. Also, the distance measurements algorithm is robust against noise. Quantization noise can be high in low lighting condition of the environment and if the distance from landmarks and camera is high.

This paper naturally opens to the development of localization algorithms based on our distance estimation algorithm. The global localization is in fact under development. Another open important issue is the landmark placement (e.g., $[23,24])$. Finally, the estimation of the orientation of the mobile object is another fundamental problem not addressed in this paper. The use of the characteristic frequencies of fluorescent lamp is an interesting method to identify the landmarks. In this case, adaptive and artificial intelligence metaphors, perhaps inherited by different scientific context (e.g., [20, 21]), may be considered. Future works will be focused on these open points.

\section{References}

[1] Sean Campbell, Niall O' Mahony, Anderson Carvalho, Lenka Krpalkova, Daniel Riordan and Joseph
Walsh, Where am I? Localization techniques for Mobile Robots A Review, 6th ICMRE International Conference, Barcelona, Spain, February 12-15, 2020, pp. $43-47$

[2] Chengyang He, Yinqiu Xia, Chengpu Yu and Chaoyang Jiang, A multi-hop distributed indoor localization algorithm for ultra-wide-band sensor network, 16th ICARCV International Conference, Shenzhen, China, December 13-15, 2020, pp.1335-1340

[3] Gaurav Kumar, Vrinda Gupta and Rahul Tank, Phasebased Angle estimation approach in Indoor Localization system using Bluetooth Low Energy, 2020 ICOSEC International Conference, Trichy, India, September 10-12, 2020, pp.904-912

[4] Glasbey, C.A., Two-dimensional generalisations of dynamic programming for image analysis, Stat Comput Vol. 19, No. 49, 2009, pp. 49-56

[5] Wei A. Shang, Survey of Nobile Robot Vision Selflocalization, Journal of Automation and Control Engineering, Vol.7, No.2, 2019, pp.98-101

[6] Anca Morar, Alin Moldoveanu, Irina Mocanu, Florica Moldoveanu, Ion Emilian Radoi, Victor Asavei, Alexandru Gradinaru and Alexandru Butean, A Comprehensive Survey of Indoor Localization Methods Based on Computer Vision, Sensors, Vol.20, No.9, 2020, pp.1-36

[7] G. Chatzimilioudis, A. Cuzzocrea, D. Gunopulos, N. Mamoulis, A novel distributed framework for optimizing query routing trees in wireless sensor networks via optimal operator placement, Journal of Computer and System Sciences 79 (3), 2013

[8] A. Cuzzocrea, S. Mansmann, OLAP visualization: models, issues, and techniques, Encyclopedia of Data Warehousing and Mining, Second Edition, 2009

[9] A. Cuzzocrea, C. De Maio, G. Fenza, V. Loia, M. Parente, OLAP analysis of multidimensional tweet streams for supporting advanced analytics, Proceedings of the 31st ACM SAC Symposium, 2016

[10] Marios Avgeris, Dimitrios Spatharakis, Nikolaos Athanasopoulos, Dimitrios Dechouniotis and Symeon Papavassiliou, Single Vision-Based Self-Localization for Autonomous Robotic Agents, 7th FiCloud International Conference, pp.123-129, 26-28 Aug., Istanbul, Turkey, 2019

[11] Zhang, C.; Zhang, X., Visible Light Localization Using Conventional Light Fixtures and Smartphones, IEEE Trans. Mob. Comput. 18, 2018, 2968-2983 
[12] Yasuo Ogawa and Joo-Ho Lee and Syunji Mori and Akira Takagi and Chie Kasuga and Hideki Hashimoto, The positioning system using the digital mark patternthe method of measurement of a horizontal distance, 1999 IEEE SMC International Conference, Tokyo, Japan, 1999, pp.731-741

[13] Alfredo Cuzzocrea and Enzo Mumolo and Daniel Pirro and Gianni Vercelli, An efficient CUDA-based approximate two-dimensional dynamic programming algorithm for advanced computer vision applications, 2016 IEEE SMC International Conference, Budapest, Hungary, October 9-12, 2016, pp.4251-4258

[14] Ming He and Chaozheng Zhu and Qian Huang and Baosen Ren and Jintao Liu, A review of monocular visual odometry, Vis. Comput., 36, 5, 2020, pp. 10531065

[15] Seiichi Uchida and Hiroaki Sakoe, A monotonic and continuous two-dimensional warping based on dynamic programming, Fourteenth ICPR International Conference, Australia, 16-20 August, 1998, pp.521524

[16] Esther Levin and Roberto Pieraccini, Dynamic planar warping for optical character recognition, 1992 IEEE ICASSP International Conference, San Francisco, California, USA, March 23-26, 1992, pp.149152

[17] Hansheng Lei and Venu Govindaraju, Direct Image Matching by Dynamic Warping, 2004 IEEE CVPR International Conference, Washington, DC, USA, June 27 - July 2, 2004

[18] K.Vinotha, Bellman Equation in Dynamic Programming, International Journal of Computing Algorithm, $05,1,2016, \mathrm{pp}=35-37$

[19] Daniel Keysers and Walter Unger, Elastic image matching is NP-complete, Pattern Recognit. Lett., 24, 1-3, 2003, pp. 445-453

[20] M. Cannataro, A. Cuzzocrea, A. Pugliese, XAHM: an adaptive hypermedia model based on XML, Proceedings of the 14th SEKE International Conference, 2002

[21] A. Cuzzocrea, Combining multidimensional user models and knowledge representation and management techniques for making web services knowledgeaware, Web Intelligence and Agent Systems: An international journal 4(3), 2006

[22] Alfredo Cuzzocrea and Luca Camilotti and Enzo Mumolo, An Innovative Monocular Mobile Object Selflocalization Approach Based on Ceiling Vision, AINA
2021 International Conference, Toronto, ON, Canada, 12-14 May, 2021, pp. 713-730

[23] Jerry Wang and Shiaofen Fang and Meie Fang and Jeremy Wilson and Noah Herrick and Susan Walsh, Automatic Landmark Placement for Large 3D Facial Image Dataset, 2019 IEEE International Conference on Big Data (Big Data), Los Angeles, CA, USA, December 9-12, 2019, pp. 5088-5093

[24] Javier Perez-Ramirez and Deva K. Borah and David G. Voelz, Optimal 3-D Landmark Placement for Vehicle Localization Using Heterogeneous Sensors, IEEE Trans. Veh. Technol., vol. 62, no. 7, 2013, pp. 29872999

[25] Jason A. Janét and Ren C. Luo and Caglan M. Aras and Michael G. Kay, Sonar windows and geometrically represented objects for mobile robot selfreferencing, Proceedings of 1993 IEEE/RSJ IROS International Conference, Tokyo, Japan, July 26 - 30, 1993, pp. 1324-1331 